\title{
Balkanologie
}

Balkanologie Revue d'études pluridisciplinaires

Vol. IX, n' 1-2 | 2005

Volume IX Numéro 1-2

\section{Seraïdari (Katerina), Le culte des icônes en Grèce}

Toulouse : Presses universitaires du Mirail, 2005, 256 pages

\section{Gilles de Rapper}

\section{(2) OpenEdition}

Journals

Édition électronique

URL : http://journals.openedition.org/balkanologie/2028

DOI : 10.4000/balkanologie.2028

ISSN : 1965-0582

Éditeur

Association française d'études sur les Balkans (Afebalk)

Édition imprimée

Date de publication : 1 décembre 2005

ISSN : 1279-7952

Référence électronique

Gilles de Rapper, «Seraïdari (Katerina), Le culte des icônes en Grèce », Balkanologie [En ligne], Vol. IX, n 1-2 | 2005, mis en ligne le 14 janvier 2010, consulté le 17 décembre 2020. URL : http://

journals.openedition.org/balkanologie/2028 ; DOI : https://doi.org/10.4000/balkanologie.2028

Ce document a été généré automatiquement le 17 décembre 2020.

(c) Tous droits réservés 


\title{
Seraïdari (Katerina), Le culte des icônes en Grèce
}

Toulouse : Presses universitaires du Mirail, 2005, 256 pages

\author{
Gilles de Rapper
}

\section{RÉFÉRENCE}

Seraïdari (Katerina), Le culte des icônes en Grèce, Toulouse : Presses universitaires du Mirail, 2005, 256 p.

1 Ce livre est une étude anthropologique du culte des icônes dans la Grèce contemporaine. L'approche anthropologique s'inspire à la fois des travaux anglosaxons sur l'anthropologie de la Grèce, principalement ceux de Michael Herzfeld et de Jill Dubisch, et des travaux sur le rituel et la mythologie chrétienne menés dans d'autres sociétés européennes par Jean-Pierre Albert, Claudine Vassas et Marlène Albert-Llorca. Il ne s'agit donc pas d'aborder l'icône comme objet mystique, à la manière des théologiens, ni comme objet esthétique à la manière des historiens de l'art, mais comme objet de dévotion, c'est-à-dire comme un produit des gestes et des paroles de dévotion. Car pour Katerina Seraïdari, ce sont les pratiques qui créent et individualisent l'icône, plus que sa signification théologique ou sa valeur esthétique: « ce n'est pas l'icône qui constitue une spécificité de la religion orthodoxe, mais ce sont les pratiques religieuses orthodoxes qui rendent l'icône spécifique » (pp. 20-21).

2 L'intérêt de l'auteur se porte donc sur les pratiques, et sur des pratiques localisées, observées à l'occasion de fêtes en divers lieux de Grèce mais principalement dans les îles. Ces observations sont complétées par l'interprétation du corpus légendaire véhiculé par les prêtres, les folkloristes et les érudits locaux comme par les fidèles et les pèlerins eux-mêmes. Cette approche comparative, mettant en évidence les variations locales dans le traitement des icônes ainsi que la multiplicité des significations accordées aux icônes et au rituel selon les acteurs et les contextes, autorise trois lectures : une lecture politique, s'intéressant aux parallèles et aux points de rencontre 
entre l'histoire locale telle qu'elle s'inscrit dans le rituel, l'histoire nationale et l'histoire sainte; une lecture spatiale, qui s'appuie sur l'analyse des pratiques de circulation des icônes entre différents espaces; une lecture en termes de genre, qui s'intéresse aux rôles différenciés des hommes et des femmes dans le rituel.

La première partie, s'appuyant sur des observations menées à Limni (Eubée), Tinos et Sifnos, montre d'abord l'importance de l'histoire sainte dans le discours de la communauté nationale comme dans celui des communautés locales: par l'intermédiaire de l'histoire sainte, les communautés locales s'inscrivent dans le temps de la nation, lui-même identifié à l'histoire sainte (« les aventures de la nation (...) sont sans cesse rapportées à la Passion ", p. 42). Le culte des icônes peut ainsi être vu comme un phénomène sociologique constitutif de la cohésion d'une communauté: "chaque communauté locale met en avant les spécificités cultuelles de ses icônes afin d'afficher sa particularité et sa cohésion, afin de créer son temps et son espace sacrés » (p. 29). Cela pose la question du rôle des hommes politiques, des intellectuels et du clergé pour donner du sens et "proposer des interprétations religieuses et politiques du passé " (p. 67). C'est l'interaction de ces discours avec les gestes dévotionnels et festifs de la communauté locale qui donne du sens aux rituels préexistants et engendre éventuellement de nouveaux rituels. L'ensemble forme ce que l'auteur appelle le «capital sacré» d'une communauté locale (p.67), capital manipulé pour affirmer la cohésion sociale de la communauté.

4 La deuxième partie s'intéresse à l'une des modalités de la «manipulation » du capital sacré, celle de la circulation et de la rotation des icônes, telle qu'elle est pratiquée dans certaines îles (Sifnos, Sikinos, Naxos). Les icônes originales ou leurs copies sont en effet soumises à des déplacements tout au long de l'année, selon des modalités différentes d'une île à l'autre. À Sifnos, l'icône patronale est transportée dans un ermitage le jour de sa fête; elle passe ensuite quinze jours dans une maison privée (qui prendra en charge les frais de la prochaine fête) avant de faire chaque jour, pendant le reste de l'année, le trajet entre cette maison où elle est gardée pendant la nuit, et l'église paroissiale où elle passe la journée. D'autres icônes circulent entre leurs églises et les maisons de fidèles qui les prennent en charge. Ces différents systèmes de rotation sont pour l'auteur des moyens de "diffusion du sacré dans le temps et dans l'espace " (p.71), ils participent à la "décentralisation» du sacré (p. 83). L'analyse permet d'aborder certaines oppositions sous-jacentes à la circulation des icônes et aux formes de dévotion dont elles sont l'objet en général: opposition entre espace domestique (celui des maisons et des chapelles privées) et espace public (églises), entre espace profane et espace sacré, entre icônes originales et copies, ces dernières pouvant se voir attribuer autant sinon plus de pouvoir en raison de leur plus grande facilité de circulation. Alors que les récits d'invention évoqués dans la première partie mettaient en évidence le rôle des icônes dans l'affirmation de la cohésion sociale des communautés locales, la circulation des icônes révèle les divisions internes de la communautés, entre paroisses ou familles, entre clergé et fidèles.

5 Dans la troisième partie, Katerina Seraïdari part d'une critique de l'association traditionnelle, en anthropologie méditerranéenne, de l'espace privé avec le "féminin » et de l'espace public avec le "masculin " pour aborder les rôles respectifs des hommes et des femmes dans les récits d'invention des icônes d'abord, puis surtout dans trois fêtes : à Nyssiros dans le Dodécanèse, à Limni en Eubée et à Ipati en Phthiotide (Grèce continentale). Une analyse très fine du rôle central ou périphérique, actif ou passif, des 
hommes et des femmes dans les différentes étapes et les différents lieux de ces fêtes permet d'aborder la construction sociale des figures féminines et masculines dans les différents contextes locaux, ainsi que leur association avec les catégories de «tradition » et de «modernité ». Les différences relevées entre les îles où les femmes semblent être moins tenues à l'écart des «structures festives» (p. 220), et la Grèce continentale où leur rôle est plus limité, sont rapidement rapportées à des différences d'organisation familiale (la résidence est généralement uxorilocale dans les îles et virilocale sur le continent), ce qui fait regretter non seulement que les structures familiales n'aient pas été prises en considération dans le reste de l'analyse mais aussi que le seul cas continental pris en compte ne permette pas d'approfondir la comparaison entre les différents systèmes coexistant en Grèce.

6 La comparaison, même interne aux seules sociétés insulaires, reste malgré tout un des points forts du livre, avec le souci de rendre compte des points de vue et des interprétations des différents acteurs: c'est la multiplicité des significations et la constante réinvention, dans le présent, des pratiques de dévotion, qui donnent aux icônes leur place centrale et en font un angle d'approche privilégié de la société grecque. 